\title{
Physical activity, airway resistance and small airway dysfunction in severe asthma
}

\author{
To the Editor:
}

Asthma is a chronic airway disease with a heterogeneous clinical presentation [1], and a subset of 5-10\% of patients suffer from severe disease [2]. Chronic obstructive pulmonary disease (COPD) is different from asthma with respect to underlying pathobiology but shares some clinical features with severe asthma, for instance fixed airway obstruction and small airway dysfunction $[3,4]$. In COPD, the consequences of the disease for physical activity in daily life have been studied intensively [5-7]; in contrast, only few data are available on patients with asthma [8]. Measurements of physical activity in adult patients with stable asthma of different severity grades are lacking, and associations with airway physiology have not been evaluated.

We aimed to measure physical activity in adult patients with mild-to-moderate and severe asthma. Furthermore, we aimed to evaluate the relationship between physical activity and lung function, including small airway dysfunction, a clinically relevant feature of asthma that might have been underestimated thus far [9].

We prospectively recruited patients with an established diagnosis of asthma and healthy controls at the Pulmonary Research Institute at LungClinic Grosshansdorf (Germany) and at the inpatient and outpatient departments of LungClinic Grosshansdorf and Research Center Borstel (Germany) between March 2014 and September 2015, after obtaining their written informed consent (local ethics approval at Medical School Luebeck, Germany; Az. 12-215).

Patients had to be in a stable phase of their disease and exclusion criteria were 1) an exacerbation or respiratory tract infection within 4 weeks prior to the study visit; and 2) presence of any severe comorbidity that could interfere with physical activity. Recruited asthma patients were classified as having either mild-to-moderate or severe asthma according to current European Respiratory Society/American Thoracic Society guidelines [2]. Patients had to be in specialist care for $>3$ months, and factors that made asthma "difficult to control" had to be addressed adequately [2]. Included asthma patients were allowed to be smokers, to avoid significant selection bias. Patients identified as smokers (i.e. either current smokers or former smokers with $\geqslant 10$ pack-years) were accurately screened for features distinguishing asthma from COPD [3], and excluded from the study if features of COPD currently prevailed [4].

We measured physical activity for 1 week using an established multisensory activity monitor (SenseWear Pro Armband; BodyMedia, Pittsburgh, PA, USA) validated for patients with COPD [5]. We analysed average steps per day (SPD) and average minutes of at least moderate activity per day (MMA; any energy expenditure $>3$ METs). We used a threshold of $94 \%$ of wearing time $(22.5 \mathrm{~h})$ to identify valid days and excluded subjects with fewer than five valid days from analyses [5].

We performed spirometry [10], body plethysmography [11] and impulse oscillometry [12], according to current guidelines. All lung function measures were performed in the morning and patients used their controller medication as usual. We studied airflow limitation using forced expiratory volume in $1 \mathrm{~s}$ (FEV1) and peak expiratory flow (PEF); airway resistance using specific effective airway resistance (sReff; body plethysmography) and resistance at $5 \mathrm{~Hz}$ (R5; impulse oscillometry); and small airway dysfunction using frequency dependence of resistance ( $f \mathrm{~d} R \mathrm{abs}(R 5-R 20)$; impulse oscillometry) [13].

We performed data analysis using the Statistical Package for Social Science (IBM SPSS Statistics version 20.0; IBM, Armonk, NY, USA). Data are presented as mean \pm SD or median (interquartile range), depending on distributional characteristics.

@ERSpublications

Physical activity is reduced in severe asthma; impulse oscillometry was a better predictor than spirometry http://ow.ly/6WdN305D9aV

Cite this article as: Bahmer T, Waschki B, Schatz F, et al. Physical activity, airway resistance and small airway dysfunction in severe asthma. Eur Respir J 2017; 49: 1601827 [https://doi.org/10.1183/ 13993003.01827-2016]. 
We enrolled 146 patients with asthma (severe asthma $n=63$; mild-to-moderate asthma $n=83$ ) and 29 healthy controls. Patients with severe asthma (mean age 55.5 years, $49 \%$ male, mean body mass index (BMI) $28.2 \mathrm{~kg} \cdot \mathrm{m}^{-2}, 22 \%$ smokers, $\mathrm{n}=24(38 \%)$ on oral corticosteroids, mean $\mathrm{FEV} 173.3 \%$ pred and median $f \mathrm{~d} R \mathrm{abs} 0.16(0.09-0.25) \mathrm{Hz})$ performed 6174 (4822-9277) SPD on average, compared to 7831 (6534-10 252) SPD in patients with mild-to-moderate asthma (mean age 48.1 years, $47 \%$ male, mean BMI $26.1 \mathrm{~kg} \cdot \mathrm{m}^{-2}, 24 \%$ smokers, mean FEV1 88.7\% pred, median $f \mathrm{~d} R$ abs $\left.0.09(0.04-0.14) \mathrm{Hz}\right)$ and $8912(6800-11$ 127) SPD in healthy controls (mean age 42.1 years, $62 \%$ male, mean BMI $24.4 \mathrm{~kg} \cdot \mathrm{m}^{-2}, 3 \%$ smokers, mean FEV 1 105.8\% pred, median $f \mathrm{~d} R \mathrm{abs} 0.03(0.02-0.05) \mathrm{Hz}$ ) (figure 1a). Average minutes of moderate activity was 125 (68-172) min in severe asthma, 151 (99-197) min in mild-to-moderate asthma and 163 (110-207) min in healthy controls. In patients with severe asthma, SPD and MMA were significantly reduced, by $21 \%$ and $17 \%$, respectively compared to mild-to-moderate asthma (ANOVA $\mathrm{p}<0.05$ ), and by $31 \%$ and $23 \%$,
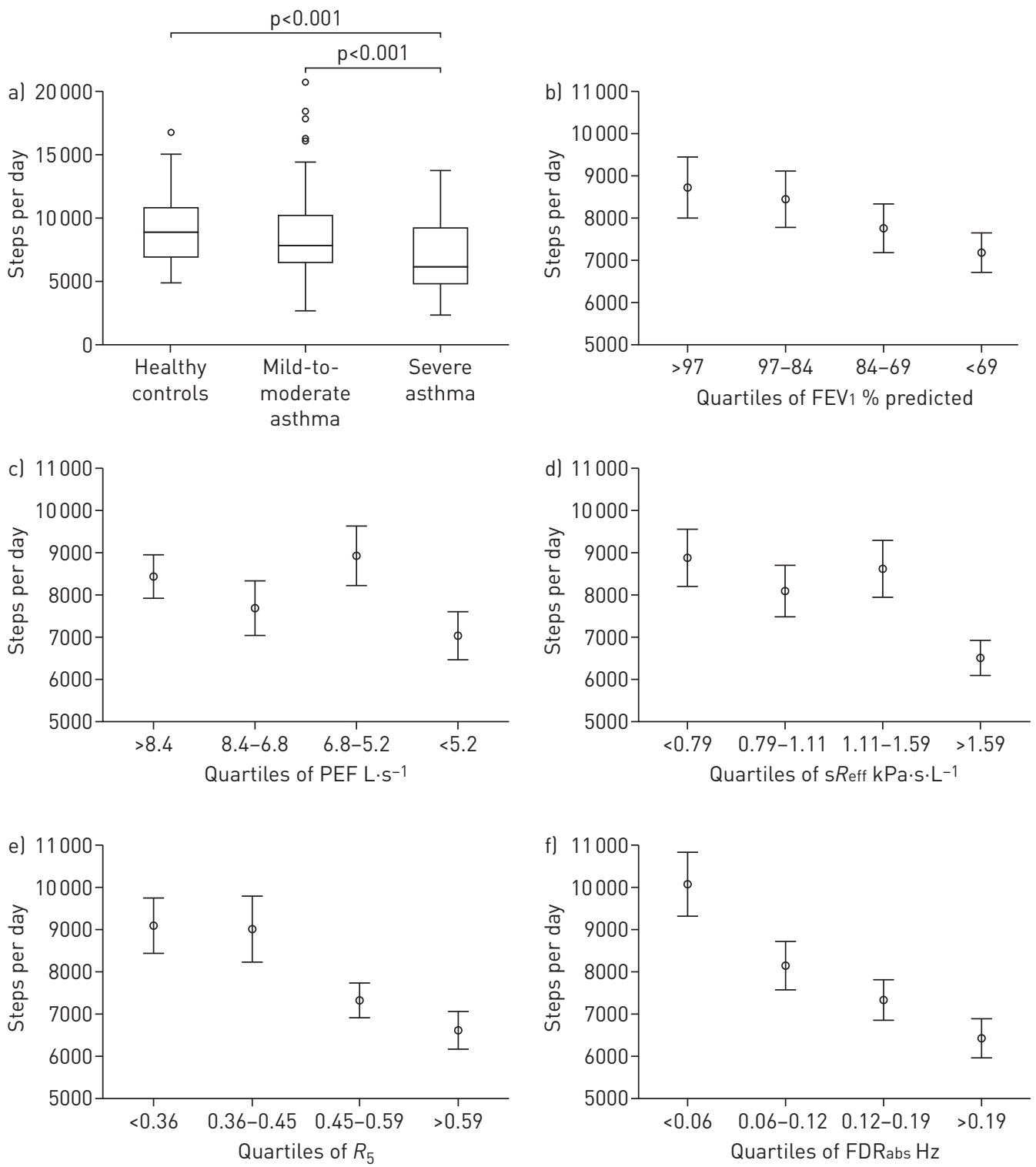

FIGURE 1 a) Number of steps per day (SPD) in healthy controls, patients with mild-to-moderate asthma and severe asthma. In unadjusted analyses (ANOVA), SPD were significantly different across groups $(p<0.001) . b-f)$ Distribution of SPD across quartiles of b) forced expiratory volume in $1 \mathrm{~s}$ (FEV $)$ \% predicted, c) peak expiratory flow (PEF), d) specific effective airway resistance (sReff), e) resistance at $5 \mathrm{~Hz}(R 5)$ and f) frequency dependence of resistance ( $\mathrm{fd}$ Rabs) in asthmatic patients. Most impaired lung function may either be represented in the lowest quartile (FEV $1 \%$ pred or PEF) or in the highest quartile (sReff, $R 5$ or $f \mathrm{~d} R a \mathrm{abs}$ ), and in both cases is displayed on the far right in each graph. Bars represent the mean \pm SE for each quartile of each lung function parameter. ANOVAs were performed to compare SPD between quartiles. In these unadjusted analyses, lowest quartile and highest quartile were significantly different for $s R$ eff $(p=0.005), R 5(p=0.003)$ and $f d R a b s(p<0.001)$. 
respectively compared to healthy controls $(\mathrm{p}<0.05)$. Multivariate regression analyses adjusting for age, sex, obesity and smoking as potential confounders of physical activity revealed that SPD were still significantly reduced in severe asthma compared to mild-to-moderate asthma (standardised coefficient $\beta-0.260$, standard error $0.068, \mathrm{p}=0.001$ ) and healthy controls (standardised coefficient $\beta-0.272$, standard error $0.095, \mathrm{p}=0.013$ ), while differences in MMA were no longer significant between groups.

In patients with asthma, distributions of SPD (median 7481 (5464-9709)) across quartiles of FEV1 (mean $82.1 \pm 20.7 \%$ pred), PEF (mean 7.0 $\pm 2.4 \mathrm{~L} \cdot \mathrm{s}^{-1}$ ), sReff (median $1.12(0.79-1.69) \mathrm{kPa} \cdot \mathrm{s} \cdot \mathrm{L}^{-1}$ ), R5 Hz (median 0.45 $\left.(0.36-0.59) \mathrm{kPa} \cdot \mathrm{s} \cdot \mathrm{L}^{-1}\right)$ and $\mathrm{f} \mathrm{d} R$ abs (median $\left.0.12(0.06-0.19) \mathrm{kPa} \cdot \mathrm{s} \cdot \mathrm{L}^{-1}\right)$, are presented in figure $1 \mathrm{~b}-\mathrm{f}$. In multivariate regression analyses adjusting for confounders (age, sex, obesity and smoking), lowest and highest quartiles could be differentiated significantly from each other for PEF, sReff, $R 5$ and $f \mathrm{~d} R$ abs (standardised coefficient $\beta$ and standard error $-0.244,0.121 ;-0.257,0.104 ;-0.295,0.106$; and -0.373 , 0.110 , respectively; $\mathrm{p}$-values $0.044,0.014,0.006$ and 0.001 , respectively), while differences in FEV 1 were not statistically significant. Differences across all quartiles (total $\mathrm{p}$-value of the whole regression model) were only significant for $R 5(\mathrm{p}=0.029)$ and $f \mathrm{~d} R \mathrm{abs}(\mathrm{p}=0.007)$.

The main findings of our study are that physical activity is reduced in severe asthma and that reduced physical activity in asthma is associated with impulse oscillometric airway resistance and small airway dysfunction, but not with airflow limitation.

Reduced levels of objectively measured physical activity in patients with difficult-to-control asthma have previously been reported by VAN 'T HUL et al. [8]. However, some of the limitation of physical activity in that study may not only be a consequence of the disease, but rather a consequence of obesity [14] and of other factors associated with difficult-to-control asthma [2]. In our population of stable, severe asthmatics, we observed a clinically meaningful difference in physical activity compared to both healthy controls and patients with mild-to-moderate asthma, indicating that severity might be a key contributor to physical activity limitation in asthma.

Most patients with severe asthma in our study had fixed airflow limitation in spirometry (i.e. FEV1/forced vital capacity ratio $<0.7$ ), a feature shared with patients with COPD [15]. The level of physical activity in patients with severe asthma in our study is comparable to that of patients with COPD with Global Initiative for Chronic Obstructive Lung Disease stage II-III in a previous study [5]. Interestingly, COPD patients in that study were $\sim 10$ years older and had more severe airflow limitation, indicating that different airway diseases may have a variable effect on daily physical activity [6], not necessarily indicated by the degree of airflow limitation.

Airflow limitation is the gold standard in lung function assessment of patients with asthma and the only marker of airway physiology reflected in the definition of severe asthma [2]. Interestingly, FEV1 and PEF were rather poor markers of physical activity in asthma in our study. In contrast, impulse oscillometric measures of airway resistance and small airway dysfunction were more closely related to daily physical activity levels. Furthermore, patients with asthma with highly abnormal $R 5$ and $f \mathrm{~d} R$ abs performed similar numbers of steps compared to patients with severe asthma and patients with normal impulse oscillometry values performed similar numbers of steps compared to healthy subjects. Interestingly, values for small airway dysfunction overlapped greatly in patients with severe asthma and mild-to-moderate asthma. This might indicate that impulse oscillometric assessment of airway resistance, and especially small airway dysfunction in a tidal breathing manoeuvre might enhance our understanding of the consequences of the disease for everyday life, irrespective of the current concept of disease severity in asthma.

Thomas Bahmer ${ }^{1,5}$, Benjamin Waschki ${ }^{1,5}$, Fee Schatz $^{2,5}$, Christian Herzmann ${ }^{3,5}$, Peter Zabel ${ }^{4,5}$, Anne-Marie Kirsten ${ }^{2,5}$, Klaus F. Rabe ${ }^{1,5}$, Henrik Watz ${ }^{2,5}$ and the ERA-Study Group ${ }^{6}$

${ }^{1}$ LungClinic Grosshansdorf, Grosshansdorf, Germany. ${ }^{2}$ Pulmonary Research Institute at LungClinic Grosshansdorf, Grosshansdorf, Germany. ${ }^{3}$ Center for Clinical Studies, Research Center Borstel, Borstel, Germany. ${ }^{4}$ Medical Clinic, Research Center Borstel, Borstel, Germany. ${ }^{5}$ Airway Research Center North (ARCN), German Center for Lung Research (DZL), Germany. ${ }^{6}$ Full details of the ERA-Study Group can be found in the Acknowledgements.

Correspondence: Thomas Bahmer, LungClinic Grosshansdorf, Woehrendamm 80, 22927 Grosshansdorf, Germany. E-mail: t.bahmer@lungenclinic.de

Received: July 142016 | Accepted after revision: Oct 182016

This study is registered at clinicaltrials.gov with identifier number NCT02419274.

Support statement: This study has been supported by the German Center for Lung Research (DZL) which is funded by governmental grants. Funding information for this article has been deposited with the Open Funder registry.

Conflict of interest: Disclosures can be found alongside this article at erj.ersjournals.com 
Acknowledgements: We thank Regine Wieland, Petra Hundack-Winter, Margret Gleiniger, Zaklina Hinz, Susann Prange, Corinna Derwort (LungClinic Grosshansdorf, Grosshansdorf, Germany), Christine Ballhausen, Johanna Döhling, Andrea Glaewe, Edda Horst, Lenka Krabbe, Birgit Kullmann, Monika Rennhack and Eva Wittmer (Research Center Borstel, Borstel, Germany) for their excellent support in study logistics.

The members of the ERA Study Group are as follows: Thomas Bahmer, Benjamin Waschki and Klaus F. Rabe (LungClinic Grosshansdorf, Pulmonology, Grosshansdorf, Germany), Anne-Marie Kirsten, Frauke Pedersen and Henrik Watz (Pulmonary Research Institute at LungClinic Grosshansdorf), Christian Herzmann and Annika Opitz (Center for Clinical Studies, Research Center Borstel, Borstel, Germany), Peter Zabel (Medical Clinic, Research Center Borstel) and Karoline I. Gaede (Biobank, Research Center Borstel).

The authors' contributions are as follows. Conception and design of the study: T. Bahmer, B. Waschki, P. Zabel, A-M. Kirsten, K.F. Rabe, H. Watz; acquisition, analysis and interpretation: T. Bahmer, B. Waschki, F. Schatz, C. Herzmann, A-M. Kirsten, H. Watz; drafting the manuscript: T. Bahmer, B. Waschki, F. Schatz, H. Watz; critically revising the manuscript: C. Herzmann, P. Zabel, A-M. Kirsten, K.F. Rabe. All authors read and approved the final version of the manuscript.

\section{References}

1 Wenzel SE. Asthma phenotypes: the evolution from clinical to molecular approaches. Nat Med 2012; 18: 716-725.

2 Chung KF, Wenzel SE, Brozek JL, et al. International ERS/ATS guidelines on definition, evaluation and treatment of severe asthma. Eur Respir J 2014; 43: 343-373.

3 Postma DS, Rabe KF. The asthma-COPD overlap syndrome. N Engl J Med 2015; 373: 1241-1249.

4 Postma DS, Reddel HK, ten Hacken NH, et al. Asthma and chronic obstructive pulmonary disease: similarities and differences. Clin Chest Med. 2014; 35: 143-156.

5 Watz H, Waschki B, Meyer T, et al. Physical activity in patients with COPD. Eur Respir J 2009; 33: 262-272.

6 Watz H, Pitta F, Rochester CL, et al. An official European Respiratory Society statement on physical activity in COPD. Eur Respir J 2014; 44: 1521-1537.

7 Waschki B, Kirsten AM, Holz O, et al. Disease progression and changes in physical activity in patients with chronic obstructive pulmonary disease. Am J Respir Crit Care Med 2015; 192: 295-306.

8 van 't Hul AJ, Frouws S, van den Akker E, et al. Decreased physical activity in adults with bronchial asthma. Respir Med 2016; 114: 72-77.

9 Contoli M, Santus P, Papi A. Small airway disease in asthma: pathophysiological and diagnostic considerations. Curr Opin Pulm Med 2015; 21: 68-73.

10 Miller MR, Hankinson J, Brusasco V, et al. Standardisation of spirometry. Eur Respir J 2005; 26: 319-338.

11 Wanger J, Clausen JL, Coates A, et al. Standardisation of the measurement of lung volumes. Eur Respir J 2005; 26: 511-522.

12 Oostveen E, MacLeod D, Lorino H, et al. The forced oscillation technique in clinical practice: methodology, recommendations and future developments. Eur Respir J 2003; 22: 1026-1041.

13 Bickel S, Popler J, Lesnick B, et al. Impulse oscillometry: interpretation and practical applications. Chest 2014; 146: 841-847.

14 Dixon AE, Poynter ME. Mechanisms of asthma in obesity. Pleiotropic aspects of obesity produce distinct asthma phenotypes. Am J Respir Cell Mol Biol 2016; 54: 601-608.

15 Contoli M, Baraldo S, Marku B, et al. Fixed airflow obstruction due to asthma or chronic obstructive pulmonary disease: 5-year follow-up. J Allergy Clin Immunol 2010; 125: 830-837. 